\title{
Re-interventions on the thoracic and thoracoabdominal aorta in patients with Marfan syndrome
}

\author{
Florian S. Schoenhoff, Thierry P. Carrel \\ Department of Cardiovascular Surgery, University Hospital Bern, Bern, Switzerland \\ Correspondence to: Florian S. Schoenhoff, MD. Department of Cardiovascular Surgery, University Hospital Bern, 3010 Bern, Switzerland. \\ Email: florian.schoenhoff@insel.ch.
}

The advent of multi-gene panel genetic testing and the discovery of new syndromic and non-syndromic forms of connective tissue disorders have established thoracic aortic aneurysms as a genetically mediated disease. Surgical results in patients with Marfan syndrome (MFS) provide an important benchmark for this patient population. Prophylactic aortic root surgery prevents acute dissection and has contributed to the improved survival of MFS patients. In the majority of patients, re-interventions are driven by a history of dissection. Patients undergoing elective root repair have a low risk for re-interventions on the root itself. Experienced centers have results after valve-sparing procedures at 10 years comparable with those seen after a modified Bentall procedure. In patients where only the ascending aorta was replaced during the initial surgery, re-intervention rates are high as the root continues to dilate. The fate of the aortic arch in MFS patients presenting with dissection is strongly correlated with the extent of the initial surgery. Not replacing the entire ascending aorta and proximal aortic arch results in a high rate of re-interventions. Nevertheless, the additional burden of replacing the entire aortic arch during emergent proximal repair is not very well defined and makes comparisons with patients undergoing elective arch replacement difficult. Interestingly, replacing the entire aortic arch during initial surgery for acute dissection does not protect from reinterventions on downstream aortic segments. MFS patients suffering from type B dissection have a high risk for re-interventions ultimately leading up to replacement of the entire thoracoabdominal aorta even if the dissection was deemed uncomplicated by conventional criteria. While current guidelines do not recommend the implantation of stent grafts in MFS patients, implantation of a frozen-elephant-trunk to create a stable proximal landing zone for future endovascular or open procedures has emerged as a means to address aortic arch and descending aortic pathologies.

Keywords: Marfan syndrome (MFS); Loeys-Dietz syndrome; aortic aneurysm; aortic surgery

Submitted Jul 03, 2017. Accepted for publication Sep 05, 2017.

doi: $10.21037 /$ acs.2017.09.14

View this article at: http://dx.doi.org/10.21037/acs.2017.09.14

\section{Introduction}

The last decade has seen an increasing awareness for patients with connective disorders and patients with Marfan syndrome (MFS) in particular. MFS has long been the only seriously considered differential diagnosis in young patients presenting with aortic aneurysms. The advent of multi-gene panel genetic testing and the discovery of new syndromic forms of connective tissue disorders such as Loeys-Dietz syndrome (LDS) as well as many forms of non-syndromic presentations have established thoracic aortic aneurysms as a genetically mediated disease (1-3). Although the spectrum has broadened, MFS still serves as a model for connective tissue disorders with vascular involvement. Surgical results in patients with MFS provide an important benchmark for other patient populations.

Low morbidity and mortality rates in patients undergoing elective root surgery have continuously lowered the threshold for surgical interventions on the proximal aorta (4). The concept of prophylactic aortic root surgery 

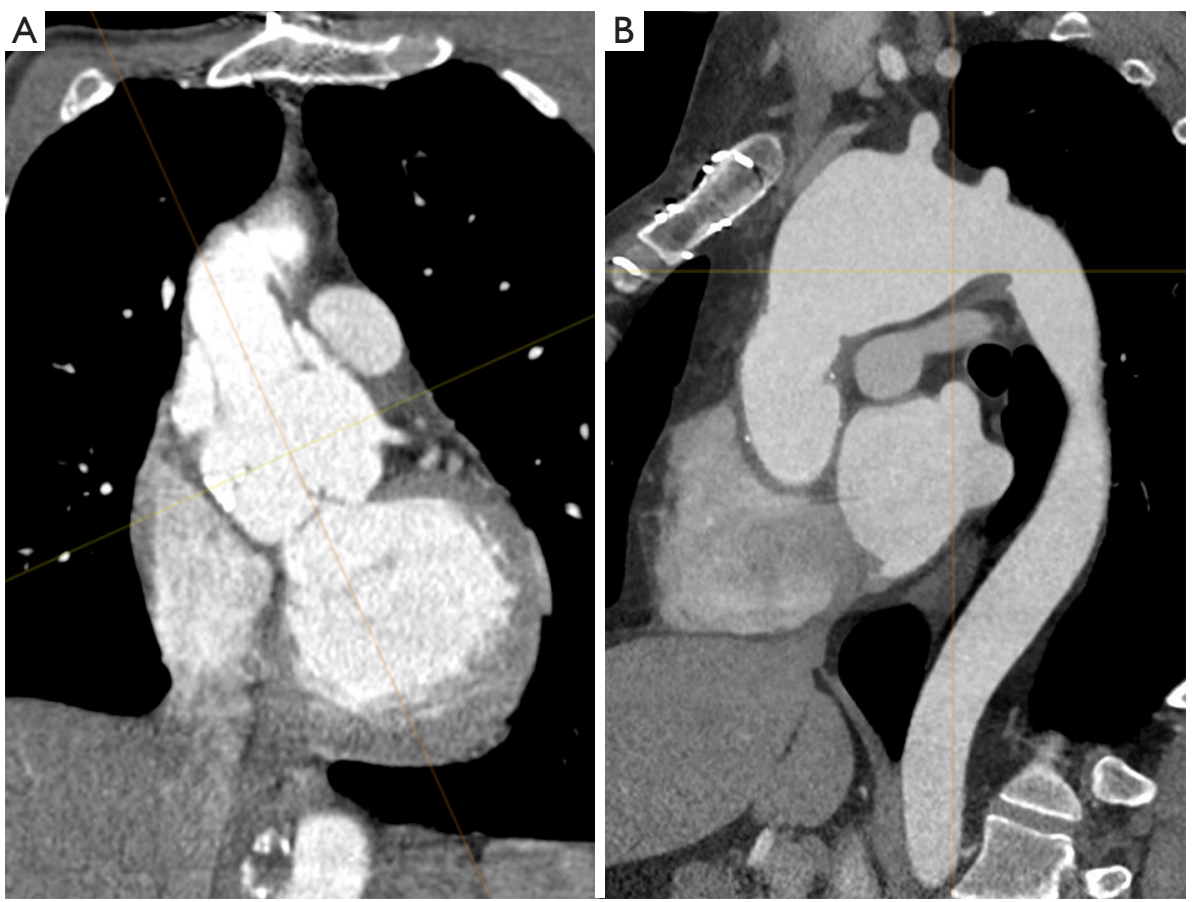

Figure 1 Reoperations of the aortic root and arch. (A) 41 y/o MFS patient with dilatation of the aortic root 2 years after replacement of the ascending aorta due to type A dissection; (B) dilatation of the aortic arch in a 34 y/o Loeys-Dietz patient 12 years after root replacement. MFS, Marfan syndrome.

in preventing acute dissection is well established (5) and has contributed to the improved survival of MFS patients over the past decades (6). Several studies suggested a shift of morbidity and mortality towards the distal aorta. Analysis of the Euro Heart Survey database revealed that $31 \%$ of interventions in patients with MFS have been performed on the distal aorta (7) and $18 \%$ of primary interventions were due to lesions on the distal aorta (8). By now there is ample evidence that aortic re-interventions in MFS are driven by a history of dissection in the majority of patients.

\section{Aortic root re-interventions}

Patients undergoing elective root repair either by a valvesparing approach using the reimplantation technique or a modified Bentall procedure have a low risk for reinterventions on the root itself. In a large study from Johns Hopkins Hospital, Baltimore, 10-year freedom from re-intervention on the aortic root after a modified Bentall procedure using a mechanical valve was $92 \%$ (9). Reasons for re-interventions on the aortic root after Bentall procedures are endocarditis or valve-dysfunction mostly due to insufficient anticoagulation. MFS patients frequently develop coronary button aneurysms, the extent of which depends on how much aortic tissue was left around the coronary ostia during the initial surgery. In our experience, re-intervention due to the sole purpose of removing coronary button aneurysms is rare. In another study by the Baltimore group representing the largest MFS cohort to date with 372 patients over a 30-year period, coronary button dehiscence was seen in three patients that underwent a classic Bentall procedure with side-to-side anastomosis of the coronaries (6).

There is a subset of MFS patients where only the ascending aorta was replaced during the initial surgery. These are mostly patients that presented with type A acute dissection where the surgeon wanted to limit the procedure and the diagnosis was not known at the time. In our experience, almost all of these patients come back for root replacement as the root continues to dilate over time and significant aortic valve insufficiency develops (Figure 1A) $(10,11)$.

As more patients are diagnosed earlier with MFS and therefore more patients are operated on earlier electively, the rate of patients that receive a valve-sparing root replacement (VSRR) is steadily increasing. While most larger series over the past 20 years report a rate of around $50 \%$ in elective cases, nowadays most of the young patients 
receive a VSRR. Nevertheless, MFS patients present with an inherent weakness of the aortic valve cusps itself that is usually not seen in other patient populations presenting with aortic root aneurysm. MFS patients frequently present with fenestrations, small tears at the commissures and elongated free margins. Although one can argue that this is just the result of stress due to the dilated aortic root and enlargement of the annulus which will cease after the root has been reconstructed, the issue of longevity of VSRR in MFS persisted. This is an important issue in adult patients where a sufficiently large mechanical prosthesis can be easily implanted and is expected to last a lifetime. Therefore, we and others, while embracing VSRR in other patient populations, have been a bit more cautious performing this procedure in patients with MFS. Due to the almost normal life-expectancy of MFS patients in the current era, the risk for thromboembolic and bleeding events due to lifelong oral anticoagulation following aortic root replacement using a mechanical valve is becoming more and more of an issue and VSRR seems an attractive option to reduce these risks. But as VSRR carries a certain risk for subsequent reintervention, decision-making in the individual patient can be challenging.

In a study comparing patients undergoing VSRR (180 patient-years) and Bentall procedures using a mechanical valve (274 patient-years) the need for re-intervention after the re-implantation procedure $(0.8 \%$ per patient-year) was not significantly higher than after a Bentall procedure. Interestingly, there was neither significant morbidity nor mortality associated with root reoperations. As with most other series, re-intervention rates after the remodeling (Yacoub) procedure were significantly higher than after the re-implantation (David) procedure. All re-interventions were performed due to progressive aortic valve insufficiency (12). The re-intervention rate after VSRR may certainly be influenced by the aggressiveness with which this approach is pursued by each center. In our practice, VSRR was adopted very early for children and adolescents but we remained hesitant in the adult population due to the inherent weakness of the aortic cusp tissue in MFS. Furthermore, many MFS patients presented at a time where the annulus was already significantly enlarged, the cusp stretched and a durable repair seemed unlikely. In our series, patients with VSRR had significantly smaller root diameters and less aortic regurgitation compared to those that underwent a Bentall procedure. The fact that there is considerable overlap indicates the importance of other parameters. Although some surgeons expressed their views that MFS itself is not a risk factor of VSRR failure anymore (13), a large international registry from the Aortic Valve Operative Outcomes in Marfan Patients Study Group found that outcome of VSRR in MFS is mostly likely different than in non-MFS patients (14). After including $316 \mathrm{MFS}$ patients from experienced centers, $7 \%$ of VSRR patients had developed grade $2 \mathrm{AR}$ after the first year, which is a worse outcome compared to non-MFS patient populations. Despite the increasing awareness for connective tissue disease, many patients are only diagnosed at a point where the aortic root size already by far exceeds the recommended threshold for intervention and thereby limits the possibility to spare the valve. In centers that maintain a more conservative approach such as Johns Hopkins Hospital, freedom-from-re-intervention after VSRR at 10 years was $93 \%$ and therefore comparable with results seen after Bentall procedures with $92 \%$ at 10 years.

Results from a large meta-analysis which analyzed 11 observational studies including 1,385 MFS patients reported a thromboembolic event rate of $0.7 \%$ per year after Bentall procedures compared to $0.3 \%$ per year after VSRR (15). Re-intervention rates were significantly higher in the VSRR group compared to the Bentall group ( $0.3 \%$ vs. $1.3 \% /$ year). But when looking only at the subgroup of patients that underwent VSRR using the re-implantation technique, the annual failure rate equals the thromboembolic event rate of $0.7 \%$ per year after Bentall procedures. In our center, the calculated annual failure rate after VSRR using the reimplantation technique is lower than the annual risk for thromboembolic or bleeding events. As the perioperative risk of re-interventions following VSRR is low, the patient might benefit from VSRR even if re-interventions may become necessary during follow-up.

\section{Aortic arch re-interventions}

As in non-MFS patients, the fate of the aortic arch in MFS patients presenting with Stanford type A aortic dissection is strongly correlated with the extent of the initial surgery. It has been clearly shown that not replacing the entire ascending aorta using hypothermic circulatory arrest results in a high rate of re-interventions. Therefore, performing a tear-oriented hemi-arch replacement with the tongueshaped prosthesis extending into the concavity of the arch is strongly recommended. Nevertheless, the additional burden of replacing the entire aortic arch as an adjunct to emergent proximal repair is not very well defined and makes comparison with patients undergoing elective total arch 


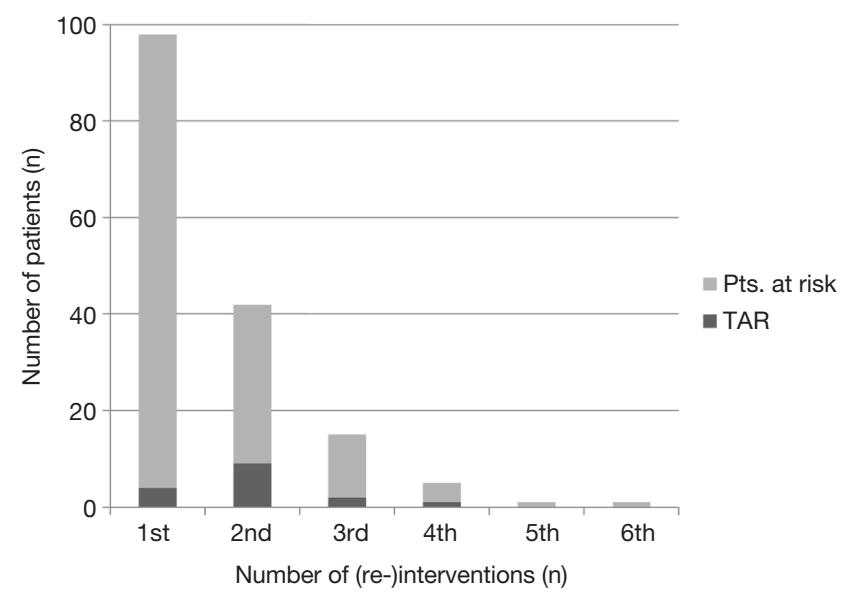

Figure 2 Graph depicting the number of total arch replacements in MFS patients compared to all re-interventions performed. TAR, total arch replacement; MFS, Marfan syndrome. Figure from Schoenhoff et al. Eur J Cardiothorac Surg 2013;44:346-51.

replacement at a later time point difficult.

Most centers reporting on outcomes after surgery for type A dissection or those dealing with re-interventions after proximal repair do not discuss arch-related morbidity and mortality separately (16-18). Even in larger series the numbers of patients with MFS undergoing aortic arch surgery is small and reports on mortality or neurologic outcome are mostly anecdotal (19). The major risk factor for the need of re-intervention on the aortic arch and distal aorta after repaired type A dissection is a patent false lumen, especially if the primary entry tear was not resected during the initial surgery. Therefore, several groups began to advocate total arch replacement and implantation of a frozen elephant trunk (FET) in addition to proximal repair in type A dissection. In a series of 44 MFS patients that underwent total arch replacement with implantation of a FET mortality was $4.8 \%$ over a mean follow-up of 3 years and only 1 neurologic event. Nevertheless, the rate of patients with chronic type A dissection was very high with $57 \%$ and most likely does not reflect general practice (20). Although primary technical success using a FET is certainly feasible, there are concerns that continued dilation of the aorta around the stent graft will limit the durability of the repair (21). There are very few reports comparing hemiarch replacement with an open distal anastomosis to total arch replacement with implantation of a frozen elephant trunk. In a large series with 120 patients presenting with acute type A dissection, mortality was $4 \%$ with no new cerebral events and a survival of $95 \%$ at 5 years in the FET and $69 \%$ in the hemi-arch group (22).

Although pseudoaneurysm or dehiscence at the level of the distal anastomosis has been described as a frequent cause for re-operation (17), it is still a rare event in our experience. Obviously, MFS patients do have a more fragile tissue and we use bovine pericardium to reinforce all anastomosis, including those of the coronary buttons. In a large series of 95 MFS patients with, $9.7 \%$ of patients required re-interventions on the distal aorta after elective aortic root surgery, compared to $44 \%$ after surgery for acute type A dissection, including $4(15 \%)$ patients with secondary total arch replacement (16).

A French group (23) reported an incidence of secondary total arch replacement of $16 \%$ after elective root surgery and of $73 \%$ in patients after type A dissection compared to $3 \%$ and $33 \%$ in our own patient population (24). We have shown that the extent of arch surgery during the initial intervention did not influence the need for thoracoabdominal repair during follow-up. This suggests that it is, again, the dissection itself that drives the need for re-operations in these patients and that the aortic arch is only one of many segments that have to be repaired over the years (Figure 1B, Figure 2) (11). In a study comparing MFS patients with and without persisting dissection after repair for type A dissection the rate for re-interventions was $50 \%$ in both groups at 10 years (19). Nevertheless, the rate of re-operation was higher in patients with a dissection in the aortic arch where only the ascending aorta was replaced compared to those patients without a dissected arch (91\% vs. $49 \%$ freedom-from-reintervention at 15 years). Replacing the aortic arch during initial surgery for AAD obviously spares the patients from secondary total arch replacement but it does not protect MFS patients from re-operations on primarily non-treated aortic segments ultimately leading up to replacement of the entire aorta. Furthermore, up to two-thirds of patients after AAD will never need additional arch procedures if the proximal arch was addressed during the initial surgery. If total arch replacement is necessary, separate re-implantation of the supra-aortic branches using a vascular graft with multiple side-branches is preferred to the island technique, in order to avoid dilatation of residual aortic tissue around the ostia of the supra-aortic vessels. Considering the advances in aortic surgery over the past decade, even complex re-operations seem to carry a moderate risk if performed in an elective setting. Therefore, delaying major additional procedures during initial surgery for acute dissection until they can be performed more safely under elective circumstances is certainly an option. 

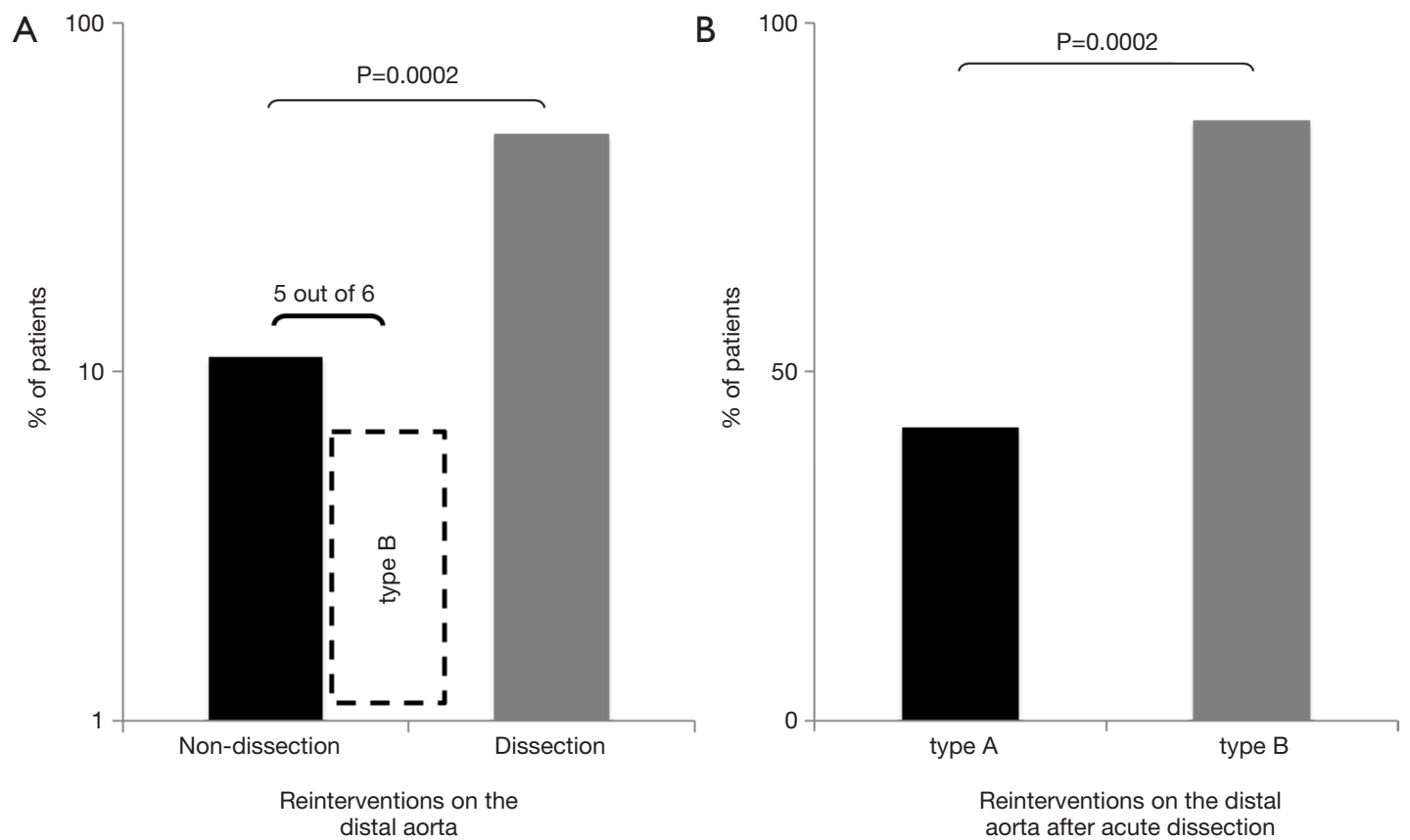

Figure 3 The rate of reinterventions on the distal aorta is significantly higher in patients with a history of acute dissection (A). Interestingly, 5 of 6 patients in the group of patients without previous dissection experienced Stanford type B dissection in the meantime. (B) High rate of patients in need for interventions on the distal aorta after type B dissection, even compared with patients with a history of type A dissection. Figure from Schoenhoff et al. Circulation 2013;16:1569-75.

\section{Thoracoabdominal aortic re-interventions}

Patients with MFS frequently must undergo interventions on the distal aorta $(8,16,25)$. In a cohort of MFS patients with a history of aortic dissection, $52 \%$ of patients experienced a clinical event, defined as new aortic dissection, surgery, ischemia, hemorrhage, within a followup period of 9.8 years (25). In our own series, re-operations on the distal aorta were significantly more frequent in patients with a history of dissection compared to those without a history of dissection (48\% vs. 11\%) (Figures 3,4). While this has already been shown to be true for patients presenting with type A dissection (44\% vs. $10 \%$ ), there is an especially high rate of re-operations (86\%) in patients with type B dissection although the descending aorta in these patients was not necessarily dilated at the time of the initial event (10). It seems that in these patients the need for subsequent surgery is driven by rapid enlargement rather than the absolute diameter. Therefore, MFS patients presenting with type $\mathrm{B}$ dissection should remain under close surveillance even if the dissection is considered uncomplicated by conventional clinical and imaging criteria (Figure 5). Type B dissection has become a substantial source of morbidity and mortality in patients with MFS. While it has been reported in the past that type B dissection represents only a small fraction of patients presenting with dissection, in our series $25 \%$ of patients presenting with acute aortic dissection suffered from type B dissection. This is in line with recently published data from a large French MFS cohort in which $20 \%$ of patients presented with type B dissection (25). It is likely that an increasing awareness of MFS leads to earlier recognition and intervention, therefore avoiding type A dissection, rather than an increase in the absolute number of type B dissections due to prophylactic proximal repair. In most patients with MFS, the aortic root slowly but constantly increases in size until dissection occurs. It is not yet clear which factors ultimately determine the moment of dissection but observational experience has shown that aortic diameter seems to be a major determinant that corresponds with the overall risk. Unfortunately, this mechanism cannot be directly transferred to type B dissection. In the IRAD database, $21 \%$ of (mostly nonMFS) patients suffering from type B dissection did not exhibit aortic dilation at the time of dissection (26), which shows that this phenomenon is not unique to MFS. While patients frequently suffer from type A dissection with aortic 


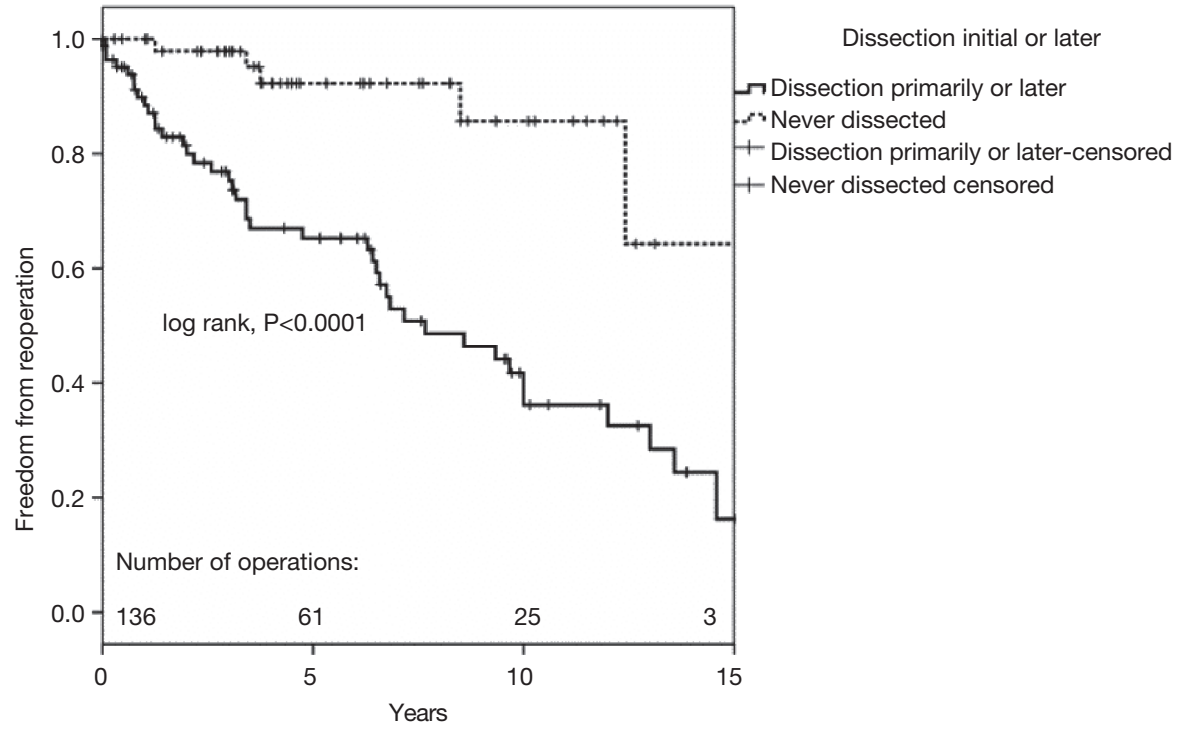

Figure 4 Kaplan-Meier curve depicting freedom-from-reoperation in patients that never experienced aortic dissection compared to those that suffered from dissection at initial presentation or during follow-up. Figure from Schoenhoff et al. Circulation 2013;16:1569-75.
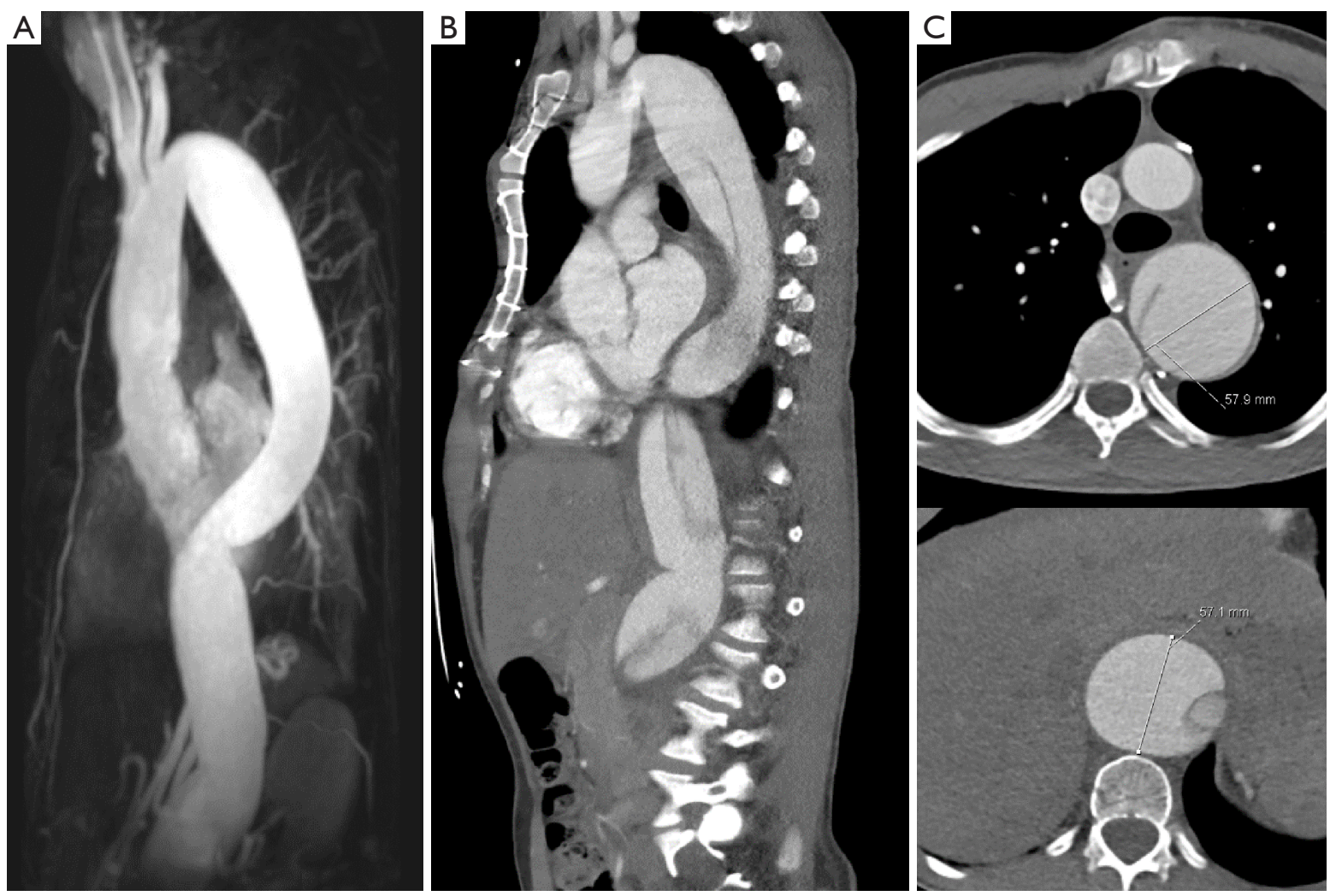

Figure 5 Stanford type B dissection in Marfan patients. (A) 26 y/o MFS patient with moderately enlarged proximal descending aorta $(40 \mathrm{~mm}) 7$ years after valve-sparing root replacement; (B) patient presents 2 weeks later with acute Stanford type B dissection; (C) rapid dilatation of the entire dissected aorta within 6 months necessitating thoracoabdominal repair. 
diameters that would have mandated surgery, this is rarely the case in MFS patients presenting with type B dissection. Although most patients with type B dissection present with a slightly enlarged proximal descending aorta, dissection occurs so infrequently at any given size that lowering thresholds for intervention would not be adequate. In the aforementioned study, patients were stratified according to size of the descending aorta ( $\leq$ or $\geq 35 \mathrm{~mm}$ ) and there was no difference in the rate of MFS patients in both groups (26). Unfortunately, so far no other risk factors apart from size have been identified, although it has been reported that pregnancy may increase the risk for type B dissection. It is difficult to estimate whether root replacement actually triggers disease progress in downstream aortic segments as has been suggested (27). Only in rare cases there seems to be a direct correlation in terms of a clamp injury or a dissection originating at the cannulation site or at the level of the distal anastomosis. Therefore, even if elective root replacement by using prosthetic materials would constitute a risk factor for downstream aortic dissection, due to the loss of the elastic properties of the root or clamp injuries of the aorta, it is obviously outweighed by the risk of type A dissection if timely proximal repair is not performed.

\section{Endovascular approaches in MFS patients}

The increasing use of endovascular approaches in the general population has certainly increased the number of MFS patients that undergo TEVAR, whether intentionally or unintentionally. While there is an abundance of case reports reporting a high rate of technical success, there is very few data on the medium- to long-term outcome of endovascular procedures in MFS patients. A 2012 joint position paper by the EACTS and the ESC states that "TEVAR is not recommended in patients with connective tissue disease except as a bail-out procedure or bridge to definitive open surgical therapy, or as a procedure following prior aortic repair when both landing zones lie within previously sited prosthetic grafts" (28). The position paper cited two references with one report on 6 MFS patients that underwent TEVAR for dissection after previous aortic root repair in 5 and solitary type B dissection in 1 patient. Followup duration was 12-74 months with 3 conversion to open surgery, 1 death and 2 patients with remodeling of the aorta $(29,30)$. The TALENT registry with 457 patients included 15 MFS patients. Median follow-up was 19.5 months (range, 1-85 months). In-hospital mortality was 5.1\%, stroke rate $3.7 \%$ and incidence of paraplegia $1.7 \%$. Unfortunately, there is no data on the outcome regarding growth of the aorta (31). In an observational study by Nordon and colleagues with seven MFS patients, aged 29 to 63 that underwent TEVAR all patients showed continued dilatation of the thoracic aorta with a median aortic growth rate of $7.2 \mathrm{~mm} /$ year (range 3.5 to 19 years) (21). This confirms our personal experience of using TEVAR in MFS patients with unsupported landing zones (Figure 6).

According to current recommendations, use of TEVAR in MFS patients should be limited to patients with both landing zones in already replaced segments of the aorta, intercostal artery patch aneurysms after thoracoabdominal repair, patients unfit for surgery or as a bridging strategy. Nevertheless, new devices and hybrid procedures somewhat blurred the lines between "endo" and "open" procedures.

In aortic surgery in general, there is a currently a trend towards addressing distal arch and descending aortic pathologies by replacing the arch using a FET and thereby creating a landing zone for subsequent TEVAR. Roselli and colleagues from the Cleveland Clinic have recently published their results of 121 out of 527 patients with connective tissue disorders with a mean follow-up of 4.4 years that underwent arch and descending aortic repair (32). In their series, $30 \%$ of first interventions on the aorta beyond the root were done using endovascular techniques, with the majority receiving TEVAR after FET. In this case, the proximal landing zone is already replaced and most probably stable. If the patients then developed type Ib endoleak, the group performed an open repair of the remaining dilated distal segment. In their series $88 \%$ of patients had MFS, 2.5\% LDS, 3.3\% Ehlers-Danlos syndrome and $5.8 \%$ were classified as "Marfanoid". This exemplifies the difficulties in correctly identifying patients with connective tissue disorders. While many patients receive specialized care once the diagnosis is made, there is a large number of patients that undergo thoracic aortic surgery without being diagnosed with a connective tissue disorders. While older data suggests that $20 \%$ of patients with thoracic aortic disease have a pathogenic mutation, more recent data suggests a much higher rate. Data from the IRAD registry has already shown that in patients presenting aortic dissection below the age of 40 years, the prevalence of MFS is as high as 50\% (33). It has been shown that $11 \%$ to $19 \%$ of patients without (known) genetic defect have at least one first degree relative with thoracic aortic disease $(34,35)$. We have retrospectively screened all patients that underwent surgery in our institution and were thought to have MFS but had a negative genetic analysis for FBN1 before the advent of testing for LDS. In this patient 

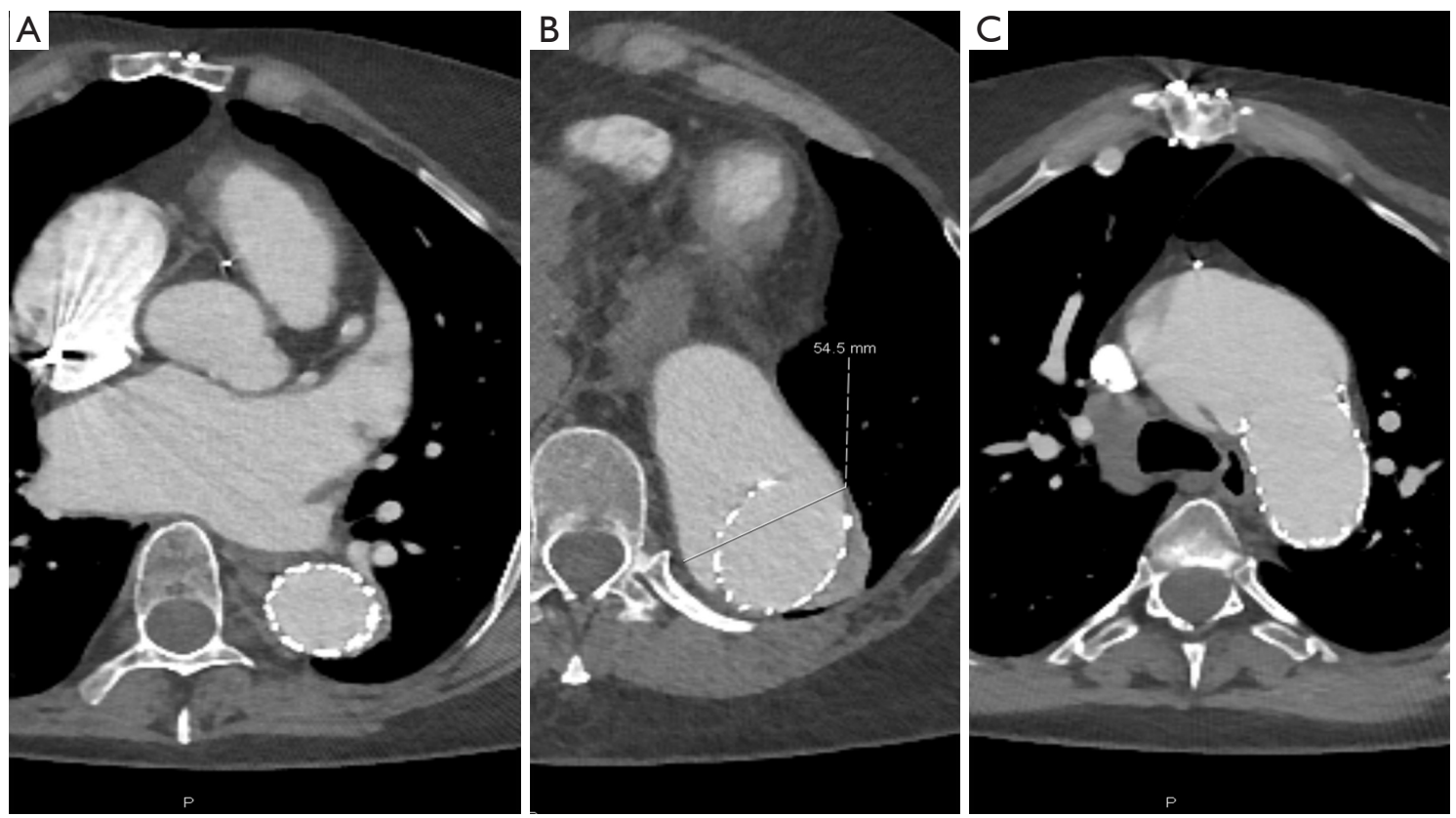

Figure 6 Endovascular repair in Marfan patients. (A) 46 y/o MFS patient with s/p TEVAR 6 years ago due to complicated type B dissection; $(\mathrm{B}, \mathrm{C})$ dilatation of the proximal and distal landing zone necessitating complete arch replacement followed by thoracoabdominal repair.

population almost $20 \%$ had a mutation that was consistent with the diagnosis of LDS (36). This shows the potential of genetic screening in this patient population. Identifying the causative mutation in patients presenting with thoracic aortic disease has a direct impact on the indication for surgery, the extent of surgery, and the prognosis of the patient, as well as his relatives.

\section{Acknowledgements}

None.

\section{Footnote}

Conflicts of Interest: The authors have no conflicts of interest to declare.

\section{References}

1. Loeys BL, Schwarze U, Holm T, et al. Aneurysm syndromes caused by mutations in the TGF-beta receptor. N Engl J Med 2006;355:788-98.

2. Loeys BL, Chen J, Neptune ER, et al. A syndrome of altered cardiovascular, craniofacial, neurocognitive and skeletal development caused by mutations in TGFBR1 or
TGFBR2. Nat Genet 2005;37:275-81.

3. van de Laar IM, Oldenburg RA, Pals G, et al. Mutations in SMAD3 cause a syndromic form of aortic aneurysms and dissections with early-onset osteoarthritis. Nat Genet 2011;43:121-6.

4. Hiratzka LF, Bakris GL, Beckman JA, et al. 2010 ACCF/AHA/AATS/ACR/ASA/SCA/SCAI/SIR/STS/ SVM guidelines for the diagnosis and management of patients with Thoracic Aortic Disease. Circulation 2010;121:e266-369.

5. Milewicz DM, Dietz HC, Miller DC. Treatment of aortic disease in patients with Marfan syndrome. Circulation 2005;111:e150-e7.

6. Cameron DE, Alejo DE, Patel ND, et al. Aortic root replacement in 372 Marfan patients: evolution of operative repair over 30 years. Ann Thorac Surg 2009;87:1344-9.

7. Engelfriet PM, Boersma E, Tijssen JG, et al. Beyond the root: dilatation of the distal aorta in Marfan's syndrome. Heart 2006;92:1238-43.

8. Finkbohner R, Johnston D, Crawford ES, et al. Marfan syndrome. Long-term survival and complications after aortic aneurysm repair. Circulation 1995;91:728-33.

9. Price J, Magruder JT, Young A, et al. Long-term outcomes of aortic root operations for Marfan syndrome: A comparison of Bentall versus aortic valve-sparing 
procedures. J Thorac Cardiovasc Surg 2016;151:330-6.

10. Schoenhoff FS, Jungi S, Czerny M, et al. Acute aortic dissection determines the fate of initially untreated aortic segments in Marfan syndrome. Circulation 2013;127:1569-75.

11. Rylski B, Bavaria JE, Beyersdorf F, et al. Type A aortic dissection in Marfan syndrome: extent of initial surgery determines long-term outcome. Circulation 2014;129:1381-6.

12. Schoenhoff FS, Langhammer B, Wustmann K, et al. Decision-making in aortic root surgery in Marfan syndrome: bleeding, thromboembolism and risk of reintervention after valve-sparing or mechanical aortic root replacement. Eur J Cardiothorac Surg 2015;48:931-5.

13. David TE, Armstrong S, Maganti M, et al. Long-term results of aortic valve-sparing operations in patients with Marfan syndrome. J Thorac Cardiovasc Surg 2009;138:859-64.

14. Coselli JS, Volguina IV, LeMaire SA, et al. Early and 1-year outcomes of aortic root surgery in patients with Marfan syndrome: a prospective, multicenter, comparative study. J Thorac Cardiovasc Surg 2014;147:1758-66.

15. Benedetto U, Melina G, Takkenberg JJ, et al. Surgical management of aortic root disease in Marfan syndrome: a systematic review and meta-analysis. Heart 2011;97:955-8.

16. Girdauskas E, Kuntze T, Borger MA, et al. Distal aortic reinterventions after root surgery in Marfan patients. Ann Thorac Surg 2008;86:1815-9.

17. Kobuch R, Hilker M, Rupprecht L, et al. Late reoperations after repaired acute type A aortic dissection. J Thorac Cardiovasc Surg 2012;144:300-7.

18. Concistrè G, Casali G, Santaniello E, et al. Reoperation after surgical correction of acute type A aortic dissection: risk factor analysis. Ann Thorac Surg 2012;93:450-5.

19. Tagusari O, Ogino H, Kobayashi J, et al. Should the transverse aortic arch be replaced simultaneously with aortic root replacement for annuloaortic ectasia in Marfan syndrome? J Thorac Cardiovasc Surg 2004;127:1373-80

20. Sun L, Li M, Zhu J, et al. Surgery for patients with Marfan syndrome with type A dissection involving the aortic arch using total arch replacement combined with stented elephant trunk implantation: the acute versus the chronic. J Thorac Cardiovasc Surg 2011;142:e85-91.

21. Nordon IM, Hinchliffe RJ, Holt PJ, et al. Endovascular management of chronic aortic dissection in patients with Marfan syndrome. J Vasc Surg 2009;50:987-91.

22. Uchida N, Shibamura H, Katayama A, et al. Operative strategy for acute type a aortic dissection: ascending aortic or hemiarch versus total arch replacement with frozen elephant trunk. Ann Thorac Surg 2009;87:773-7.

23. Bachet J, Larrazet F, Goudot B, et al. When should the aortic arch be replaced in Marfan patients? Ann Thorac Surg 2007;83:S774-9.

24. Schoenhoff FS, Kadner A, Czerny M, et al. Should aortic arch replacement be performed during initial surgery for aortic root aneurysm in patients with Marfan syndrome? Eur J Cardiothorac Surg 2013;44:346-51.

25. Mimoun L, Detaint D, Hamroun D, et al. Dissection in Marfan syndrome: the importance of the descending aorta. Eur Heart J 2011;32:443-9.

26. Trimarchi S, Jonker FH, Froehlich JB, et al. Acute type B aortic dissection in the absence of aortic dilatation. J Vasc Surg 2012;56:311-6.

27. Scharfschwerdt M, Sievers HH, Greggersen J, et al. Prosthetic replacement of the ascending aorta increases wall tension in the residual aorta. Ann Thorac Surg 2007;83:954-7.

28. Grabenwöger M, Alfonso F, Bachet J, et al. Thoracic Endovascular Aortic Repair (TEVAR) for the treatment of aortic diseases: a position statement from the European Association for Cardio-Thoracic Surgery (EACTS) and the European Society of Cardiology (ESC), in collaboration with the European Association of Percutaneous Cardiovascular Interventions (EAPCI). Eur J Cardiothorac Surg 2012;42:17-24.

29. Ince H, Rehders TC, Petzsch M, et al. Stent-grafts in patients with marfan syndrome. J Endovasc Ther 2005;12:82-8.

30. Cooper DG, Walsh SR, Sadat U, et al. Treating the thoracic aorta in Marfan syndrome: surgery or TEVAR? J Endovasc Ther 2009;16:60-70.

31. Fattori R, Nienaber CA, Rousseau H, et al. Results of endovascular repair of the thoracic aorta with the Talent Thoracic stent graft: the Talent Thoracic Retrospective Registry. J Thorac Cardiovasc Surg 2006;132:332-9.

32. Roselli EE, Idrees JJ, Lowry AM, et al. Beyond the Aortic Root: Staged Open and Endovascular Repair of Arch and Descending Aorta in Patients With Connective Tissue Disorders. Ann Thorac Surg 2016;101:906-12.

33. Januzzi JL, Isselbacher EM, Fattori R, et al. Characterizing the young patient with aortic dissection: results from the International Registry of Aortic Dissection (IRAD). J Am Coll Cardiol 2004;43:665-9.

34. Coady MA, Davies RR, Roberts M, et al. Familial patterns of thoracic aortic aneurysms. Arch Surg 1999;134:361-7. 
35. Biddinger A, Rocklin M, Coselli J, et al. Familial thoracic aortic dilatations and dissections: a case control study. J Vasc Surg 1997;25:506-11.

36. Schoenhoff FS, Mueller C, Czerny M, et al. Outcome

Cite this article as: Schoenhoff FS, Carrel TP. Reinterventions on the thoracic and thoraco-abdominal aorta in patients with Marfan syndrome. Ann Cardiothorac Surg 2017;6(6):662-671. doi: 10.21037/acs.2017.09.14 of aortic surgery in patients with Loeys-Dietz syndrome primarily treated as having Marfan syndrome. Eur J Cardiothorac Surg 2014;46:444-9. 